\title{
Maternal nutrition and fetal growth: the role of iron status and intake during pregnancy
}

\author{
This article was published in the following Dove Press journal: \\ Nutrition and Dietary Supplements \\ 5 July 2012 \\ Number of times this article has been viewed
}

\author{
CL Rodriguez-Bernal ${ }^{1,3}$ \\ M Rebagliato ${ }^{2,3}$ \\ F Ballester ${ }^{1,3,4}$ \\ 'Centro Superior de Investigacion \\ en Salud Publica (Center for Public \\ Health Research), ${ }^{2}$ Direccion \\ General de Salud Publica, \\ Generalitat Valenciana (Public \\ Health Board, Valencia), Valencia, \\ Spain; ${ }^{3}$ Consortium for Research \\ on Epidemiology and Public Health \\ (CIBERESP), ${ }^{4}$ University of Valencia, \\ Valencia, Spain
}

Correspondence: Clara L RodriguezBernal

Centro Superior de Investigacion en Salud Publica, Avenida Cataluna 21,

Valencia 46020, Spain

Tel +34961 925909

Fax +34 96I 925703

Email rodriguez_claber@gva.es

\begin{abstract}
Fetal growth restriction is an important predictor of infant mortality and morbidity. Maternal iron status and iron supplementation during pregnancy have been related to fetal growth with contrasting results. Therefore, indication of iron supplementation, doses, and timing is still controversial. Current literature was reviewed in PubMed/MEDLINE using the following terms: "iron" or "iron deficiency" or "anemia" or "iron intake" or "iron supplementation" and "pregnancy" or "maternal" and "fetal growth" or "birth weight" or "pregnancy outcomes" or "birth outcomes." The search was limited to studies in humans since 1991 assessing iron status or supplementation in the periconceptional period or at any time during pregnancy. Evidence suggests that iron deficiency in early pregnancy has an adverse effect on fetal growth. On the other hand, high levels of maternal iron markers have shown a deleterious effect at different stages of pregnancy. The mechanisms underlying such effect are unclear. It is important to acknowledge the need to assess markers of iron status adequately in order to reach reliable conclusions regarding their relationship with fetal growth. In regard to supplementation, it has been shown that low or moderate doses of iron in early pregnancy have a positive effect on fetal growth, regardless of maternal iron status. No such effect has been seen in trials of supplementation later in pregnancy, with low or high doses. Nevertheless, results should be interpreted with caution as some randomized controlled trials lack adequate methodology. Studies assessing the effect of iron supplementation in early pregnancy on fetal growth in iron-deficient and iron-sufficient women are needed in order to establish the most appropriate indications for doses and timing.
\end{abstract}

Keywords: iron, birth weight, pregnancy outcomes, nutrition, review, supplementation

\section{Introduction}

Fetal growth restriction is an important predictor of children's immediate and future health. Fetuses who do not reach their full growth potential or babies who are born low-weight have an increased risk of infant mortality and morbidity, ${ }^{1}$ including poor cognitive and neurological development, ${ }^{2}$ and a higher risk of developing chronic diseases in adulthood, ${ }^{3}$ such as coronary heart disease and type 2 diabetes. ${ }^{4-6}$ Optimal fetal growth is dependent on receiving an adequate maternoplacental nutrient supply. Thus, maternal nutrition has a critical role in fetal development and evidence suggests that fetal growth is most vulnerable to maternal dietary deficiencies of nutrients during early pregnancy. ${ }^{7}$

Iron deficiency is the most widespread nutrient deficiency in the world. ${ }^{8}$ Women of reproductive age and particularly pregnant women are at high risk for iron deficiency. ${ }^{8}$ Although such deficiency is a problem mainly in developing countries, ${ }^{9}$ it also affects 
an important percentage of women of reproductive age $(10 \%-30 \%)$ in the industrialized world $;^{10,11}$ one of the reasons being the low intake of iron from diet. ${ }^{12-15}$ This problem can be aggravated in gravid women because during pregnancy iron demand is augmented in order to cover the needs of the increase in the red cell mass, the expansion of the plasma volume, and to allow for the growth of the fetoplacental unit. ${ }^{16}$ It has been established that iron demands increase mainly during the second and third trimesters of pregnancy. ${ }^{17,18}$ As a consequence, recommendations on universal supplementation starting at the second trimester of pregnancy have been made. ${ }^{19,20}$ However, the maternal iron status at different times during pregnancy or even preconceptionally has been linked to different pregnancy outcomes, including fetal growth. ${ }^{21-23}$ It has also being suggested that under certain conditions, supplementation might have an adverse effect on pregnancy outcomes. ${ }^{24}$ Some authors recommend supplementation schemes to be tailored according to the initial nutritional status of the mother-to-be. ${ }^{25}$ Thus, indication of iron supplementation, doses, and timing is still controversial, especially among women who are not iron deficient. ${ }^{12,25}$

This article will therefore examine the relationship between maternal iron status or intake and fetal growth by conducting a descriptive review of recent evidence. The focus will be on the effect of low and high maternal levels of iron - as measured by serum markers - and intake from supplements at different times during pregnancy. This paper will present the included studies and their main results, examine the possible pathways linking maternal iron status or intake with fetal growth, summarize main findings from evidence available regarding serum iron markers, as well as dose and timing of supplementation, and also suggest areas in which future research is needed. Considering the diversity of exposure and outcomes presented in this work, an appendix with definitions of the terms used (regarding both iron status and fetal growth) is included to facilitate interpretation for readers.

\section{Methods}

\section{Design}

This paper is a descriptive review of epidemiological studies.

\section{Search strategy and selection criteria}

A search of studies was conducted on PubMed/MEDLINE. The medical subject heading (MeSH) descriptors or free text terms used were: "iron" or "iron deficiency" or "anemia" or "iron intake" or "iron supplementation" and "pregnancy" or "maternal" and "fetal growth" or "birth weight" or "pregnancy outcomes" or "birth outcomes." Furthermore, using the references of the works selected, reference harvesting technique was used. The search was limited to articles published in English.

Observational studies and randomized controlled trials (RCTs) that reported pregnancy outcomes related to fetal growth were included. The search was limited to studies in human subjects since 1991. The studies of interest were those that assessed iron status or supplementation in the periconceptional period or at any time during pregnancy and in which the measurements of fetal growth outcomes had been performed immediately after birth. Editorials and duplicated works were excluded. Studies in animals were retained for their usefulness when presenting the possible mechanisms of action underlying the relationship between iron status or intake and fetal growth.

\section{Studies assessing the relationship between iron status or supplement intake and fetal growth}

Several observational studies and RCTs trying to elucidate the association between iron maternal status or intake and fetal growth have been published, ranging from the assessment of iron status preconceptionally to studying the effect of supplementation in women without iron deficiency.

\section{Deficient maternal iron status and fetal growth}

Studies assessing the association between deficient maternal iron status - as measured by markers such as hemoglobin or serum ferritin levels - and fetal growth have examined such relationships at different times during pregnancy in diverse populations. Table 1 presents an overview of the studies linking inadequate iron status previous to, or during pregnancy, with fetal growth considered in the present review. The results are described below in more detail.

A study that assessed preconceptional iron status (measuring hemoglobin or serum ferritin levels) among Chinese textile workers likely to become pregnant found associations with several fetal growth indicators at birth. ${ }^{22}$ Preconceptional anemia was associated with reductions in birth weight, birth length, and head circumference. Infants of women with moderate anemia were $192 \mathrm{~g}$ lighter and had around four and six times higher risks of low birth weight (LBW) and fetal growth restriction, respectively, when compared to nonanemic women. Low prepregnancy ferritin concentration was associated with lower birth weight (106 g) only. ${ }^{22}$ 


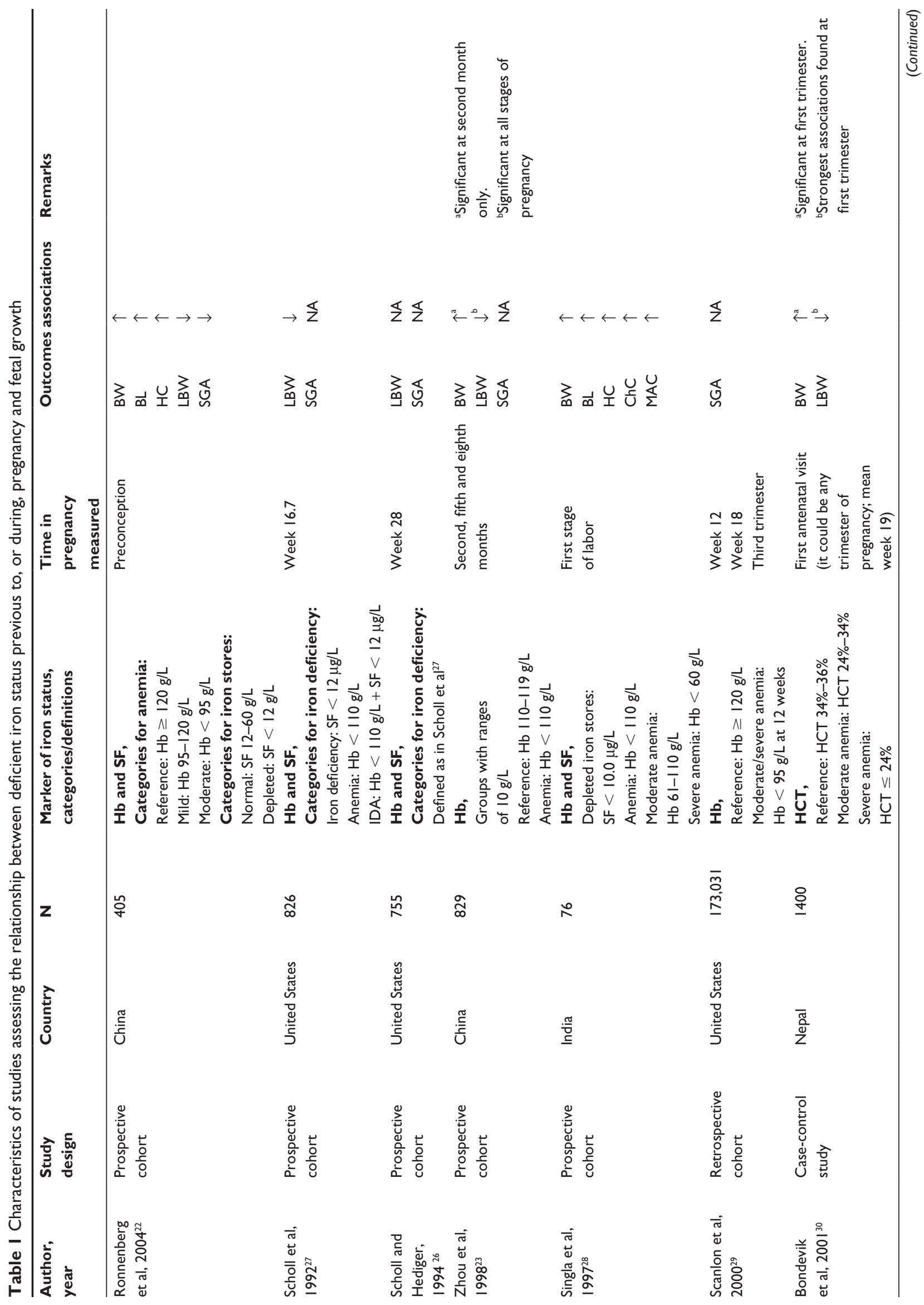




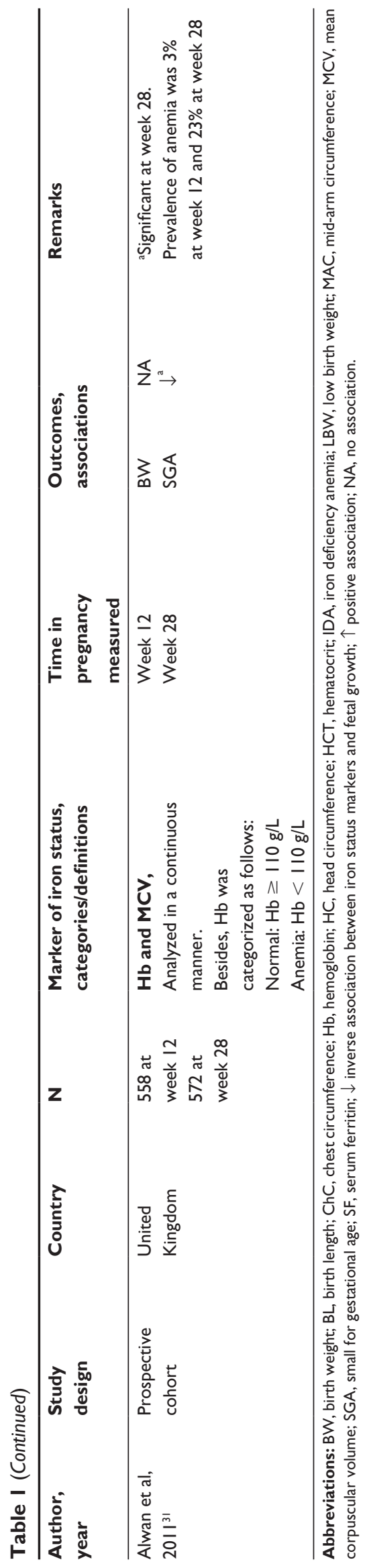

Scholl and Hediger, and Scholl et al studied the relationship between iron deficiency anemia in early and late pregnancy and fetal growth in a cohort of women belonging to a minority in the United States. ${ }^{26,27}$ Iron deficiency anemia was associated with a greater risk of LBW in early but not late pregnancy. In Chinese pregnant women, rates of LBW were higher and the risk of LBW tripled among women who were anemic during the first, second, and third trimesters of pregnancy compared to nonanemic women. ${ }^{23}$ However, mean birth weight increased with a rise in hemoglobin levels at first trimester only. Similarly, a case-control study in Nepal reported a significant increase in birth weight with increasing maternal hematocrit value recorded in the first trimester, but not during the second or third trimesters. ${ }^{30}$ Women with severe anemia at all stages of pregnancy doubled the risk of delivering LBW infants; when stratifying according to trimester of pregnancy, the association was found to be stronger for severe anemia in the first trimester with a 15 -fold increase in the risk. ${ }^{30}$ Results from a study in India also suggested a negative effect of anemia during pregnancy on different anthropometric measurements at birth. ${ }^{28}$ Contrary to these results, two studies in different populations (United States and United Kingdom) did not find a relationship between iron status markers (hemoglobin or mean corpuscular volume [MCV]) at different stages of pregnancy and fetal growth outcomes. ${ }^{29,31}$ It is important to consider that in the British study only $3 \%$ of the sample had hemoglobin levels under $11 \mathrm{~g} / \mathrm{dL}$ at 12 weeks.

It has been suggested that the relationship between maternal anemia and a higher risk of adverse pregnancy outcomes may depend on the period of pregnancy in which the anemia is measured, ${ }^{16}$ which is consistent with several of the published studies considered in this review. Evidence supports the hypothesis that anemia or deficient iron status in early pregnancy, or even in the preconceptional period, is a risk factor for impaired fetal growth. However, some studies have also shown an adverse effect of anemia or deficient iron status in mid or late pregnancy. The relationship between serum indicators of deficient iron status in mid and late pregnancy and fetal growth needs to be further studied. Moreover, it is important to acknowledge that hemoglobin and MCV do not represent specific or sensitive measures of body iron stores as they are only affected when iron deficiency is pronounced. Serum ferritin is the most reliable marker of iron status, ${ }^{32,33}$ however, it has been used only in half of the studies assessing iron deficiency considered in the present work. ${ }^{22,26-28}$ It is important to bear in mind that conditions different to iron deficiency might affect levels of iron markers. 
For example, under chronic infections, there is inhibition of erythrocytes production, but it must be differentiated from ferropenic anemia. If the infectious process produces hemolysis, then hemoglobin levels decrease due to destruction of red blood cells. Besides, conditions such as infection, inflammation, or neoplasia might result in an increase of serum ferritin concentration and could mask a state of iron deficiency. Furthermore, in late pregnancy, serum ferritin levels decrease even when bone marrow iron is present. ${ }^{20}$ It is thus necessary to set up studies which include adequate indicators of deficient iron status in order to reach reliable conclusions regarding fetal growth, especially before frank anemia develops.

At the population level, the use of multiple indicators would be a useful way of assessing iron status. The best combination would be hemoglobin, serum transferrin receptors, and serum ferritin or bone marrow iron, which would reflect functional impairment, tissue avidity for iron, and iron storage, respectively. Nevertheless, this approach is controversial as the possibility of measuring several indices to determine iron status might not be feasible in resource-poor settings. ${ }^{20}$ Besides, it has been stated that the use of multiple indicators only partially overcomes the limitation of using a single parameter. ${ }^{57}$

\section{High levels of iron markers in serum and fetal growth}

Many of the studies which evaluated the effect of deficient maternal iron status on birth weight, and have been mentioned previously in this review, also assessed the effect of high levels of iron status indicators on fetal growth. Characteristics of those and additional studies (selected for this review) linking high serum levels of iron markers previous to, or during pregnancy and fetal growth are presented in Table 2.

Ronnenberg et al found that high ferritin concentrations in the periconceptional period were related to both a reduction in birth weight (123 g) and an almost three-fold risk of small gestational age (SGA). The relationship was stronger than that found with deficient iron status. ${ }^{22}$ Previous and subsequent studies, have also shown such relationship. A study including British women of different ethnicities found a U-shaped relationship between hemoglobin concentration and birth outcome. ${ }^{34}$ The greatest negative association, however, was found for high rather than low hemoglobin concentration. A gradual increase in the risk of poor outcome was found with higher levels of hemoglobin reaching a seven-fold increase in the rates of LBW in women with the highest hemoglobin levels compared to the reference category. Results were similar for all ethnic groups. ${ }^{34}$ Scanlon et al showed that women from the United States with high hemoglobin levels at the first and second trimesters of pregnancy had a higher risk of giving birth to SGA infants. ${ }^{29}$ Zhou et al, in a study including Chinese pregnant women, found a trend towards an increased risk of LBW with high hemoglobin measures in all trimesters of pregnancy; however, these results did not reach statistical significance. ${ }^{23}$ A prospective study including pregnant Indian women found that maternal serum ferritin at late but not mid gestation was inversely related to various anthropometric measures at birth. ${ }^{35}$ Similarly, a prospective study in the United Kingdom found that higher hemoglobin in late but not early pregnancy was significantly associated with higher risk of SGA. ${ }^{31}$

Most of the studies considered in the present review have shown an inverse relationship between fetal growth and high levels of maternal iron markers at different stages of pregnancy in diverse population groups. The mechanisms underlying such association are unclear. It is important to bear in mind that conditions such as infection, inflammation, or neoplasia might result in an increase of serum ferritin concentration. Therefore, interpretation of serum ferritin levels is problematic in populations in which, with the exception of parasitic infections and malaria, the incidence of infection or inflammation is high. ${ }^{20}$

\section{Intake of iron supplements and fetal growth}

Several RCTs have been conducted in order to assess the effect of iron supplementation at different times during pregnancy on fetal growth in diverse population groups. Different doses and schemes of supplementation have been reported. Table 3 summarizes the RCTs considered in this review. Results are described below in more detail.

Two RCTs conducted in industrialized countries, one in Norway ${ }^{36}$ and another in Australia, ${ }^{37}$ among nonanemic women who were assigned a low-dose iron supplement or placebo from week 20 of pregnancy onwards found that supplementation had a positive effect on maternal iron status. Nevertheless, no significant differences were found in anthropometry at birth (birth weight, birth length, or head circumference) between the iron-supplemented and placebo groups. Small sample size makes the interpretation of the results in the Norwegian study difficult. An RCT in which adolescent and adult pregnant women from the United States participated also assessed the effect of iron supplements on pregnancy outcomes in iron-sufficient women. ${ }^{38}$ Participants were assigned to receive either $60 \mathrm{mg}$ of elemental iron 


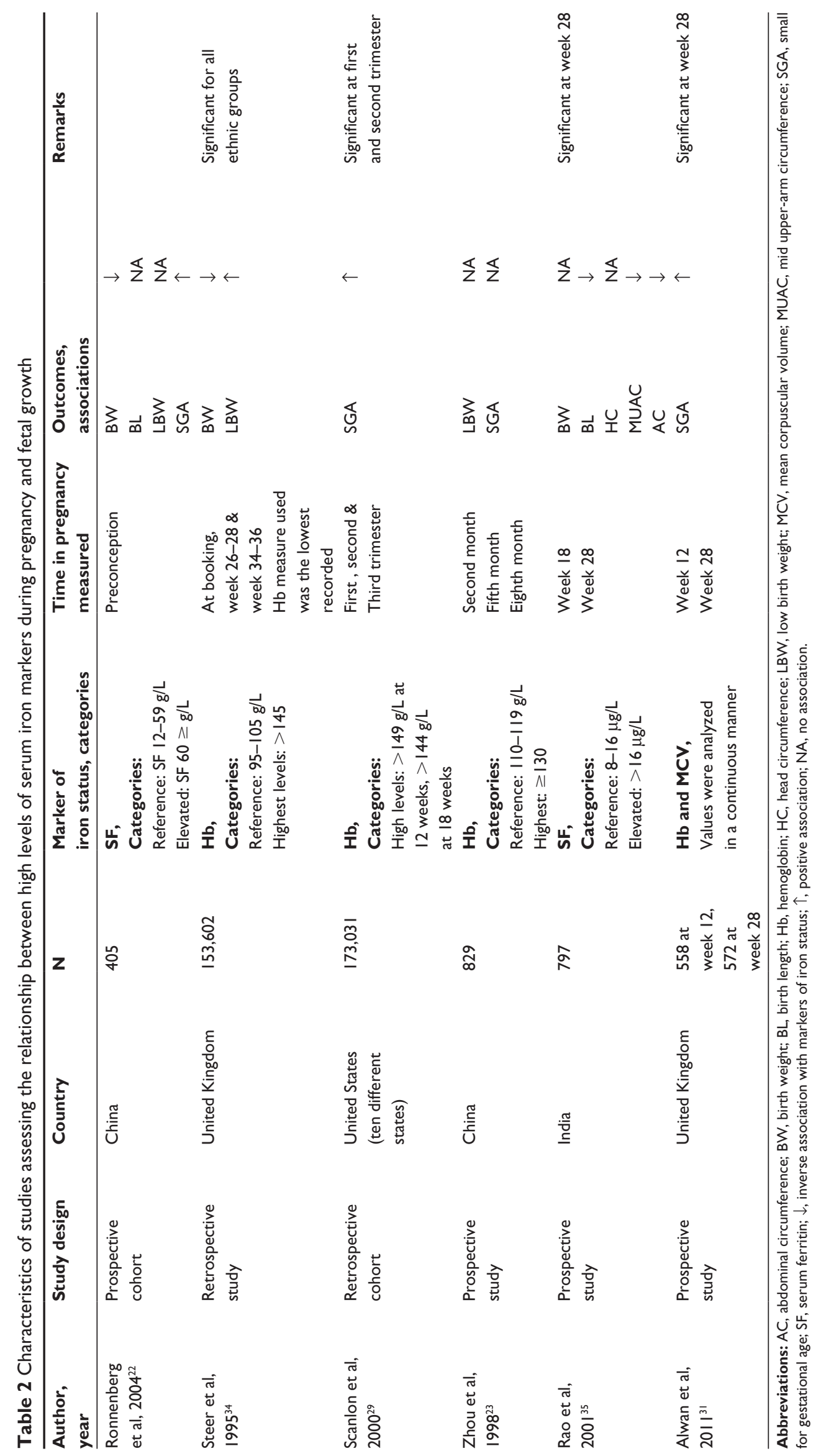




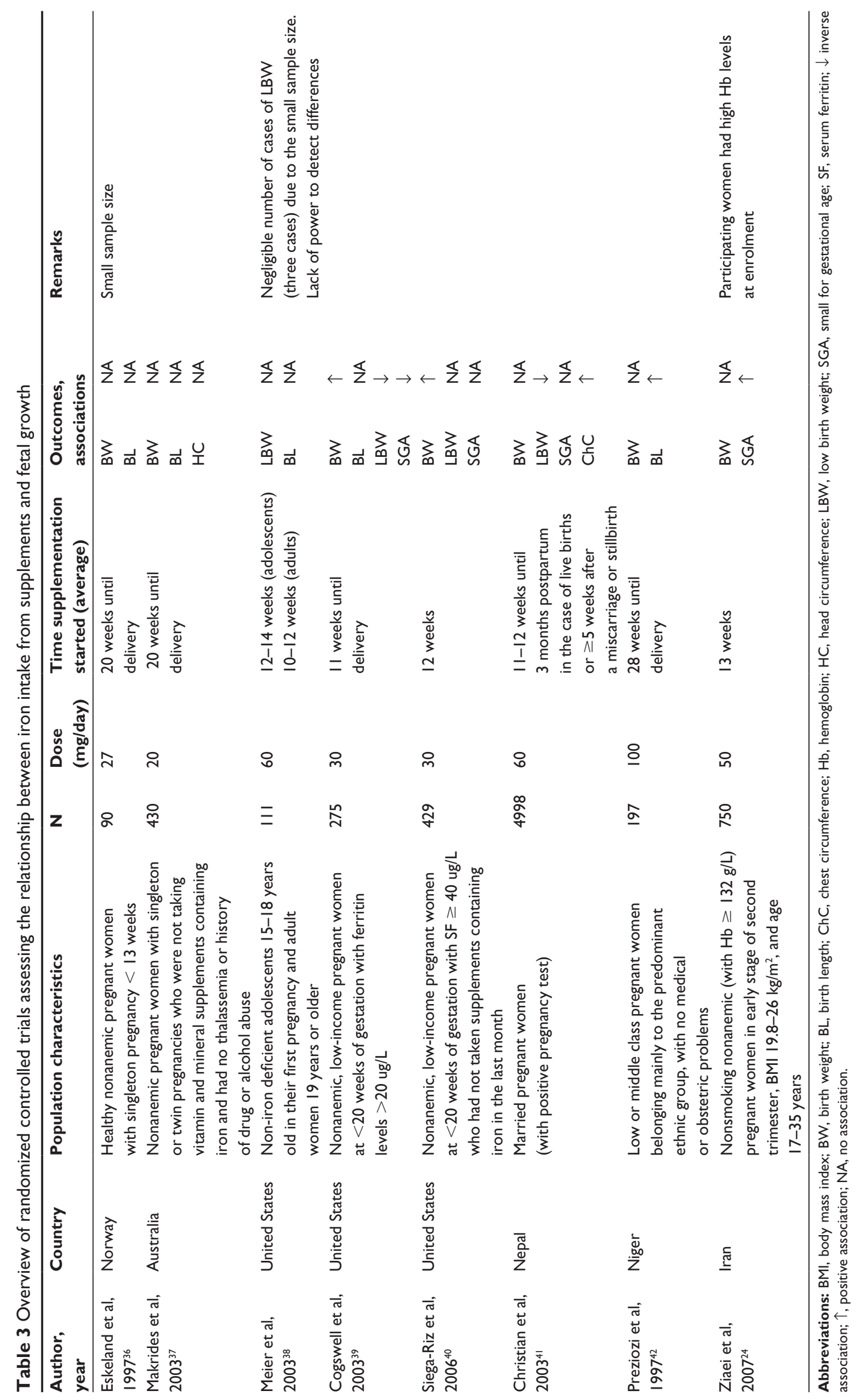


or placebo from the beginning of the second trimester of pregnancy until delivery. Iron supplementation reduced the proportion of iron deficiency anemia during pregnancy but did not have an effect on fetal growth outcomes. The negligible number of cases of LBW (three cases), due to the small sample size, prevents any meaningful conclusion from being drawn. Two studies conducted in the United States among low-income women had the same characteristics: all the subjects were nonanemic and iron replete at the time they were assigned to treatment; from week eleven, on average, to week 28 of gestation, women received supplementation with iron or placebo; and from week 28 onwards, women received iron supplements, as recommended by the established protocol, according to their iron status at that moment. ${ }^{39,40}$ Results were also very similar. No significant differences were found in either study between groups in any of the iron status indicators measured; however, mean birth weight increased in iron-supplemented women in both studies and the incidence of SGA decreased in one of them. ${ }^{39,40}$

In rural Nepal, where prevalence of iron deficiency anemia is high (around 40\%), an RCT was conducted. ${ }^{41}$ It included five treatment groups (around 650 pregnant women with results for anthropometry at birth). Only three arms were relevant for this review. The control group received vitamin A. Women assigned to the other two groups were given, besides vitamin A, folic acid or folic acid plus iron. The supplementation started at 11-12 weeks of pregnancy and was given until delivery. Folic acid plus iron supplementation, but not folic acid alone, significantly increased chest circumference by $0.35 \mathrm{~cm}$ and reduced the incidence of LBW by $16 \%$, so the positive effect on growth was attributed to iron. No significant effects were found on the proportion of infants who were SGA. An RCT in Niger studied pregnant women from 28 weeks of gestation until delivery. ${ }^{42}$ Around $60 \%$ of women were anemic when supplementation started. Women in the intervention group were given a high-dose supplement and those in the control group were given placebo. Birth length was significantly higher in the ironsupplemented group. No differences were found regarding birth weight. A study was conducted to determine the effect of iron supplementation on pregnancy outcome in Irani women with hemoglobin $\geq 13.2 \mathrm{~g} / \mathrm{dL}$ at the beginning of the second trimester. ${ }^{24}$ Women allocated to the case group were given $50 \mathrm{mg} /$ day of elemental iron (women in the control group were given placebo) throughout pregnancy. All the participants received only $1 \mathrm{mg}$ of folic acid daily, and they were not permitted to consume any other preparations with vitamins and other minerals. Rates of SGA were significantly higher in the case group in comparison with the control group ( $15.7 \%$ versus $10.3 \%$, respectively). There were no significant differences in mean birth weight between the two groups.

In summary, studies starting supplementation in mid or late pregnancy (low/moderate doses in high-income settings), although related to an increase in maternal iron markers, found no effect on fetal growth; ${ }^{36,38}$ the exception being one study with high-dose supplementation in a low-income setting that showed a positive association with birth length but not with birth weight. ${ }^{42}$ On the contrary, those starting supplementation in early pregnancy (low doses in highincome settings or moderate doses in low-income settings), showed a beneficial effect on fetal growth but did not seem to modify maternal iron status. ${ }^{39-41}$ The only RCT finding a negative association with fetal growth was conducted among women with relatively high hemoglobin levels at the time the intervention started. ${ }^{24}$ Results should be interpreted with caution due to methodological issues of some of the studies considered here (ie, small sample sizes).

\section{Potential mechanisms in the link between iron and fetal growth Iron deficiency}

Various biological mechanisms have been proposed to explain the pathways through which iron deficiency, including its more severe form, anemia, could impair fetal growth. Maternal and fetal stress can result from the increase of norepinephrine concentrations generated by iron deficiency or hypoxia resulting from anemia.

Maternal and fetal stress, in turn, activates the production of corticotropin-releasing hormone which stimulates fetal cortisol. As a result, longitudinal growth of the fetus could be impaired by the action of cortisol. ${ }^{43}$ Animal studies support this hypothesis. ${ }^{44-46}$ There is evidence from studies in rats of the stress caused by iron deficiency, showed by the rise in cortisol concentrations as a result of an iron-free diet. ${ }^{44}$ Besides, cortisol has been found to regulate fetal growth in sheep through physiologically decelerating growth in late pregnancy and lowering the rate of normal growth when infused in early pregnancy. ${ }^{45}$ Cortisol has also been found to inhibit fetal insulin-like growth factor 2 production, which is involved in the fetal growth process. ${ }^{46}$

Another plausible hypothesis is that erythrocytes and the fetoplacental unit might undergo oxidative damage caused by iron deficiency. ${ }^{43}$ Lastly, maternal infections - the likelihood of which increases with iron deficiency - can prompt the production of corticotropin-releasing hormone; again, the 
production of cortisol could have a negative effect on fetal growth. ${ }^{43}$

\section{Increased levels of iron markers in maternal plasma and fetal growth}

High levels of iron status markers have been associated with adverse pregnancy outcomes in several studies at different times during the pregnancy period. It has been suggested that high levels of hemoglobin or serum ferritin reflect a failure in adequate plasma volume expansion or increased blood viscosity as a result of macrocytosis which would impair uteroplacental blood flow. This, in turn, might adversely affect fetal growth. ${ }^{29,34}$ This hypothesis, however, would not explain the relationships between high hemoglobin in very early pregnancy or the periconceptional period and adverse pregnancy outcomes found in some studies ${ }^{22,23}$ as at these stages, changes in the maternal plasma volume have not occurred. Chronic inflammation has been proposed as the cause of the rise in serum ferritin levels in one study. ${ }^{22}$ That is a plausible explanation in that population of textile workers, but it would be difficult to generalize it to other populations which are not exposed to agents producing an inflammatory response. Taking into account that ferritin is a stored form of iron, an alternative interpretation of such findings is that a lower serum ferritin concentration reflects the successful transfer of iron, making it available for fetal growth..$^{33}$ There is evidence of an inverse relationship between maternal serum ferritin at the third trimester of pregnancy and the net amount of the oral iron tracer present in the neonates' circulation (which represents maternofetal iron transfer). ${ }^{48}$ Maternal iron absorption has been found to be positively correlated with the amount of iron in the newborn's serum, ${ }^{47}$ which would support such hypothesis.

\section{Effect of intake of iron supplements on fetal growth}

Based on the evidence linking deficient maternal iron status in early pregnancy with impaired fetal growth, the finding that low- or moderate-dose iron supplementation in early pregnancy benefits fetal growth in women with deficient iron status is not surprising. The fact that supplementation in early pregnancy did not seem to have an effect on maternal serum iron markers but did have a positive effect on fetal growth could be explained by a preferential transfer of iron to the placenta and fetus; ${ }^{39}$ evidence suggests that dietary iron is transferred to the fetus rapidly before incorporation in maternal hemoglobin. ${ }^{47}$ The mechanisms for preferential transfer of iron are still unclear.
It has been proposed that iron supplements given late in pregnancy might cause a temporary iron overload, ${ }^{49}$ which could explain the increase in serum iron concentrations found in the studies where supplementation started later in pregnancy. It might be possible that the doses given in those trials were not high enough to produce oxidative stress ${ }^{49}$ and therefore fetal growth was not affected. Studies supplementing rats at high doses (equivalent to $120 \mathrm{mg}$ /day in humans) showed a continuous absorption of supplemental iron which was evidenced by excessive liver iron levels..$^{50,51}$ Evidence of oxidative stress was also observed in the animals. ${ }^{52,53}$ Supplementation with moderate doses in women with already high iron markers could have a similar effect and might explain the negative association found in one RCT between iron supplementation and fetal growth.

\section{Conclusion}

Evidence suggests that iron deficiency in early pregnancy has an adverse effect on fetal growth. On the other hand, elevated levels of iron serum markers during pregnancy have been negatively associated with fetal growth. It has been proposed to be the result of a failure in adequate plasma volume expansion or of a chronic inflammatory process, or a reflection of an inadequate transfer of stored maternal iron to the fetus. It is important to acknowledge the need to assess markers of iron status adequately in order to reach reliable conclusions regarding their relationship with fetal growth.

The effect of iron supplementation during pregnancy on fetal growth has been studied in diverse population groups with varying results. It has been shown that low or moderate dose supplementation in early pregnancy has a positive effect on fetal growth in women with both adequate and deficient iron status. No such effect has been seen in trials of supplementation later in pregnancy, with either low or high doses, although some studies lack adequate methodology to draw definite conclusions. Supplementation in pregnant women with high hemoglobin levels was found to be detrimental for fetal growth; nevertheless, evidence regarding this aspect is scarce.

Studies assessing the effect of iron supplementation in early pregnancy or the periconceptional period on fetal growth and other pregnancy outcomes in iron-deficient and iron-sufficient women are needed in order to establish if supplementation should be universal or tailored according to the baseline nutritional status/profile of the mother-to-be and to determine the best time to start it, if necessary. In any case, it is necessary to find the best strategies to improve the intake of nutrient-rich foods in women of childbearing age 
in order to ensure women enter pregnancy with an adequate nutritional status that helps them to cope with the demands gestation involves.

\section{Acknowledgments}

The authors are grateful to Mrs Maria Luz Bernal (BSN) and Dr Hernan Ulloa (Internal Medicine physician) from Hospital San Rafael (Facatativa, Colombia) for their useful comments and advice regarding clinical implications of the present review.

\section{Disclosure}

The authors report no conflicts of interest in this work. This study had no external funding source.

\section{References}

1. McIntire DD, Bloom SL, Casey BM, Leveno KJ. Birth weight in relation to morbidity and mortality among newborn infants. $N$ Engl J Med. 1999;340(16):1234-1238.

2. Richards M, Hardy R, Kuh D, Wadsworth ME. Birthweight, postnatal growth and cognitive function in a national UK birth cohort. Int J Epidemiol. 2002;31(2):342-348.

3. Barker DJ, Eriksson JG, Forsen T, Osmond C. Fetal origins of adult disease: strength of effects and biological basis. Int J Epidemiol. 2002;31(6):1235-1239.

4. Barker DJ. The fetal origins of coronary heart disease. Acta Paediatr Suppl. 1997;422:78-82.

5. Barker DJ. Adult consequences of fetal growth restriction. Clin Obstet Gynecol. 2006;49(2):270-283.

6. Eriksson JG. Early growth, and coronary heart disease and type 2 diabetes: experiences from the Helsinki Birth Cohort Studies. Int JObes (Lond). 2006;30 Suppl 4:S18-S22.

7. Wu G, Bazer FW, Cudd TA, Meininger CJ, Spencer TE. Maternal nutrition and fetal development. J Nutr. 2004;134(9):2169-2172.

8. Ramakrishnan U. Prevalence of micronutrient malnutrition worldwide. Nutr Rev. 2002;60(5 Pt 2):S46-S52.

9. Viteri FE. Prevention of iron deficiency. In: Howson CP, Kennedy E, Horwitz A, editors. Micronutrient Deficiencies: A Toolkit for Policymakers and Public Health Workers. Washington, DC: National Academy Press; 1998:45-102.

10. Hercberg S, Preziosi P, Galan P. Iron deficiency in Europe. Public Health Nutr. 2001;4(2B):537-545.

11. Looker AC, Dallman PR, Carroll MD, Gunter EW, Johnson CL. Prevalence of iron deficiency in the United States. JAMA. 1997;277(12): 973-976.

12. Sukchan P, Liabsuetrakul T, Chongsuvivatwong V, Songwathana P, Sornsrivichai V, Kuning M. Inadequacy of nutrients intake among pregnant women in the deep south of Thailand. BMC Public Health. 2010;10:572.

13. Pathak P, Kapil U, Kapoor SK, et al. Prevalence of multiple micronutrient deficiencies amongst pregnant women in a rural area of Haryana. Indian J Pediatr. 2004;71(11):1007-1014.

14. Turner RE, Langkamp-Henken B, Littell RC, Lukowski MJ, Suarez MF. Comparing nutrient intake from food to the estimated average requirements shows middle- to upper-income pregnant women lack iron and possibly magnesium. J Am Diet Assoc. 2003;103(4):461-466.

15. Erkkola M, Karppinen M, Järvinen A et al. Folate, vitamin D, and iron intakes are low among pregnant Finnish women. Eur J Clin Nutr. 1998;52:742-748.

16. Scholl TO. Iron status during pregnancy: setting the stage for mother and infant. Am J Clin Nutr. 2005;81(5):1218S-1222S.
17. Viteri FE. The consequences of iron deficiency and anemia in pregnancy. In: Allen L, King J, Lonnerdal B, editors. Nutrient Regulation During Pregnancy, Lactation and Growth. New York, NY: Plenum Press; 1994:127-139.

18. Bothwell TH. Iron requirements in pregnancy and strategies to meet them. Am J Clin Nutr. 2000;72(Suppl 1):257S-264S.

19. Stoltzfus RJ, Dreyfuss ML. Guidelines for the Use of Iron Supplements to Prevent and Treat Iron Deficiency Anemia. Washington, DC: International Nutritional Anemia Consultative Group; 1998.

20. World Health Organization. Iron Deficiency Anaemia: Assessment, Prevention, and Control. Geneva: World Health Organization; 2001.

21. Allen LH. Anemia and iron deficiency: effects on pregnancy outcome. Am J Clin Nutr. 2000;71(Suppl 5):1280S-1284S.

22. Ronnenberg AG, Wood RJ, Wang X, et al. Preconception hemoglobin and ferritin concentrations are associated with pregnancy outcome in a prospective cohort of Chinese women. J Nutr. 2004;134(10):2586-2591.

23. Zhou LM, Yang WW, Hua JZ, Deng CQ, Tao X, Stoltzfus RJ. Relation of hemoglobin measured at different times in pregnancy to preterm birth and low birth weight in Shanghai, China. Am J Epidemiol. 1998;148(10):998-1006.

24. Ziaei S, Norrozi M, Faghihzadeh S, Jafarbegloo E. A randomised placebo-controlled trial to determine the effect of iron supplementation on pregnancy outcome in pregnant women with haemoglobin $>$ or $=13.2 \mathrm{~g} / \mathrm{dL}$. BJOG. 2007;114(6):684-688.

25. Milman N. Iron prophylaxis in pregnancy - general or individual and in which dose? Ann Hematol. 2006;85(12):821-828.

26. Scholl TO, Hediger ML. Anemia and iron-deficiency anemia: compilation of data on pregnancy outcome. Am J Clin Nutr. 1994;59(Suppl 2):492S-501S.

27. Scholl TO, Hediger ML, Fischer RL, Shearer JW. Anemia vs iron deficiency: increased risk of preterm delivery in a prospective study. Am J Clin Nutr. 1992;55(5):985-988.

28. Singla PN, Tyagi M, Kumar A, Dash D, Shankar R. Fetal growth in maternal anaemia. J Trop Pediatr. 1997;43(2):89-92.

29. Scanlon KS, Yip R, Schieve LA, Cogswell ME. High and low hemoglobin levels during pregnancy: differential risks for preterm birth and small for gestational age. Obstet Gynecol. 2000;96(5 Pt 1):741-748.

30. Bondevik GT, Lie RT, Ulstein M, Kvale G. Maternal hematological status and risk of low birth weight and preterm delivery in Nepal. Acta Obstet Gynecol Scand. 2001;80(5):402-408.

31. Alwan NA, Greenwood DC, Simpson NA, McArdle HJ, Godfrey KM, Cade JE. Dietary iron intake during early pregnancy and birth outcomes in a cohort of British women. Hum Reprod. 2011;26(4):911-919.

32. Milman N. Iron and pregnancy - a delicate balance. Ann Hematol. 2006;85(9):559-565.

33. Milman N, Byg KE, Bergholt T, Eriksen L, Hvas AM. Body iron and individual iron prophylaxis in pregnancy - should the iron dose be adjusted according to serum ferritin? Ann Hematol. 2006;85(9):567-573.

34. Steer P, Alam MA, Wadsworth J, Welch A. Relation between maternal haemoglobin concentration and birth weight in different ethnic groups. BMJ. 1995;310(6978):489-491.

35. Rao S, Yajnik CS, Kanade A, et al. Intake of micronutrient-rich foods in rural Indian mothers is associated with the size of their babies at birth: Pune Maternal Nutrition Study. J Nutr. 2001;131(4):1217-1224.

36. Eskeland B, Malterud K, Ulvik RJ, Hunskaar S. Iron supplementation in pregnancy: is less enough? A randomized, placebo controlled trial of low dose iron supplementation with and without heme iron. Acta Obstet Gynecol Scand.1997;76(9):822-828.

37. Makrides M, Crowther CA, Gibson RA, Gibson RS, Skeaff CM. Efficacy and tolerability of low-dose iron supplements during pregnancy: a randomized controlled trial. Am J Clin Nutr. 2003;78(1):145-153.

38. Meier PR, Nickerson HJ, Olson KA, Berg RL, Meyer JA. Prevention of iron deficiency anemia in adolescent and adult pregnancies. Clin Med Res. 2003;1(1):29-36.

39. Cogswell ME, Parvanta I, Ickes L, Yip R, Brittenham GM. Iron supplementation during pregnancy, anemia, and birth weight: a randomized controlled trial. Am J Clin Nutr. 2003;78(4):773-781. 
40. Siega-Riz AM, Hartzema AG, Turnbull C, Thorp J, McDonald T, Cogswell ME. The effects of prophylactic iron given in prenatal supplements on iron status and birth outcomes: a randomized controlled trial. Am J Obstet Gynecol. 2006;194(2):512-519.

41. Christian P, Khatry SK, Katz J, et al. Effects of alternative maternal micronutrient supplements on low birth weight in rural Nepal: double blind randomised community trial. BMJ. 2003;326(7389):571.

42. Preziosi P, Prual A, Galan P, Daouda H, Boureima H, Hercberg S. Effect of iron supplementation on the iron status of pregnant women: consequences for newborns. Am J Clin Nutr. 1997;66(5):1178-1182.

43. Allen LH. Biological mechanisms that might underlie iron's effects on fetal growth and preterm birth. J Nutr. 2001;131(2S-2):581S-589S.

44. Campos MS, Barrionuevo M, Alferez MJ, et al. Interactions among iron, calcium, phosphorus and magnesium in the nutritionally iron-deficient rat. Exp Physiol. 1998;83(6):771-781.

45. Fowden AL, Szemere J, Hughes P, Gilmour RS, Forhead AJ. The effects of cortisol on the growth rate of the sheep fetus during late gestation. $J$ Endocrinol. 1996;151(1):97-105.

46. Li J, Saunders JC, Gilmour RS, Silver M, Fowden AL. Insulin-like growth factor-II messenger ribonucleic acid expression in fetal tissues of the sheep during late gestation: effects of cortisol. Endocrinology. 1993;132(5):2083-2089

47. O'Brien KO, Zavaleta N, Abrams SA, Caulfield LE. Maternal iron status influences iron transfer to the fetus during the third trimester of pregnancy. Am J Clin Nutr. 2003;77(4):924-930.

48. Roughead ZK, Hunt JR. Adaptation in iron absorption: iron supplementation reduces nonheme-iron but not heme-iron absorption from food. Am J Clin Nutr. 2000;72(4):982-989.

49. Casanueva E, Viteri FE. Iron and oxidative stress in pregnancy. J Nutr. 2003;133(5 Suppl 2):1700S-1708S.

50. Viteri FE, Liu X, Martin A, Tolomei K. True absorption and retention of supplemental iron is more efficient when administered every three days rather than daily to iron-normal and iron-deficient rats. $J$ Nutr. 1995;125(1):82-91.

51. Srigiridhar K, Nair KM, Subramanian R, Singotamu L. Oral repletion of iron induces free radical mediated alterations in the gastrointestinal tract of rat. Mol Cell Biochem. 2001;219(1-2):91-98.
52. Srigiridhar K, Nair KM. Supplementation with alpha-tocopherol or a combination of alpha-tocopherol and ascorbic acid protects the gastrointestinal tract of iron-deficient rats against iron-induced oxidative damage during iron repletion. Br J Nutr. 2000;84(2):165-173.

53. Beutler E. Disorders of iron metabolism. In: Lichtman MA, Beutler E, Kipps TJ, et al, editors. Williams Hematology, 7th ed. New York, NY: McGraw-Hill; 2006:511

54. Cook JD. Diagnosis and management of iron-deficiency anaemia. Best Pract Res Clin Haematol. 2005;18(2):319-332.

55. Expert Scientific Working Group. Summary of a report on assessment of the iron nutritional status of the United States population. Am J Clin Nutr. 1985;42(6):1318-1330.

56. Bothwell TH, Charlton RW, Cook JD, Finch CA. Iron Metabolism in Man. Oxford: Blackwell Scientific Publications; 1979.

57. World Health Organization/United Nations Children's Fund/United Nations University. Indicators for assessing iron deficiency and strategies for its prevention. Geneva: World Health Organization; 1996.

58. Walters GO, Miller FM, Worwood M. Serum ferritin concentration and iron stores in normal subjects. J Clin Pathol. 1973;26(10):770-772.

59. Milman N. Serum ferritin in Danes: studies of iron status from infancy to old age, during blood donation and pregnancy. Int J Hematol. 1996;63(2):103-135.

60. Hill CC, Pickinpaugh J. Physiologic changes in pregnancy. Surg Clin North Am. 2008;88(2):391-401.

61. Mandruzzato G, Antsaklis A, Botet F, et al. Intrauterine restriction (IUGR). J Perinat Med. 2008;36(4):277-281.

62. Imdad A, Yakoob MY, Siddiqui S, Bhutta ZA. Screening and triage of intrauterine growth restriction (IUGR) in general population and high risk pregnancies: a systematic review with a focus on reduction of IUGR related stillbirths. BMC Public Health. 2011;11 Suppl 3:S1.

63. Gardosi J, Figueras F, Clausson B, Francis A. The customised growth potential: an international research tool to study the epidemiology of fetal growth. Paediatr Perinat Epidemiol. 2011;25(1):2-10.

64. Kramer MS. Determinants of low birth weight: methodological assessment and meta-analysis. Bull World Health Organ. 1987;65(5):663-737. 


\section{Appendix: definition of terms Related to iron status} Iron deficiency

Iron deficiency, similarly to other micronutrient deficiencies, develops over time. Different levels of iron deficiency have been established:

1. Iron depletion, ie, the stage at which iron stores in the body are significantly reduced or absent as indicated by lowered values of serum ferritin. However, blood hemoglobin levels are usually normal, and iron deficiency is generally undetected by hemoglobin or hematocrit screening. ${ }^{53}$ Although evidence on the adverse consequences of an absence of iron stores is not conclusive, a rapid decrease in the level of functional compounds might occur when iron balance is compromised. ${ }^{10}$

2. Iron deficient erythropoiesis, ie, the stage at which there is a shortage of iron available so the requirement of the erythroid marrow in order to synthesize hemoglobin is not fully met. This is associated with an elevation of erythrocyte protoporphyrin and serum transferrin receptor levels. ${ }^{54}$ Hemoglobin levels are usually reduced, but they may still be above the established cutoff values for anemia. ${ }^{55}$ However, with time, frank anemia develops. ${ }^{10} \mathrm{In}$ iron deficient erythropoiesis, storage iron may be normal or even increased due to impaired release of iron into the circulation. ${ }^{54}$

3. Iron deficiency anemia is the most severe form of iron deficiency, characterized by a reduction in hemoglobin which produces distortion of red cells, leading to microcytosis and hypochromia. ${ }^{8,10,56}$ Recommended cutoff values for hemoglobin levels used to define anemia in female populations are: $110 \mathrm{~g} / \mathrm{L}$ in nonpregnant women and $120 \mathrm{~g} / \mathrm{L}$ in pregnant women; however, such levels vary by age, race, and physiologic status as well as altitude and smoking status. ${ }^{57}$ Besides, cutoff values also vary according to the stage of pregnancy. Anemia is defined as hemoglobin of $<110 \mathrm{~g} / \mathrm{L}$ in the first and third trimester and $<105 \mathrm{~g} / \mathrm{L}$ in the second trimester.

\section{Markers of iron status}

\section{Hemoglobin}

Hemoglobin is the iron-containing oxygen-transport metalloprotein in red blood cells. Due to the simplicity and low cost of the analysis, besides being recommended by the World Health Organization, ${ }^{57}$ hemoglobin concentrations are widely used as a proxy marker of iron deficiency; however, serum hemoglobin levels are usually unaltered during the initial phases of iron depletion and iron deficiency is generally undetected by hemoglobin or hematocrit screening. ${ }^{53}$ This makes hemoglobin an unsuitable marker to assess iron status. ${ }^{32}$

\section{Serum ferritin}

Ferritin is a protein in which intracellular iron is stored. Serum ferritin levels represent mobilizable iron reserves in the organism and therefore are a good biomarker for iron status. ${ }^{58,59}$

\section{Hematocrit}

Hematocrit is the percentage of erythrocyte concentration in blood. During pregnancy (towards the end of the first trimester), plasma volume increases as an adaptive physiologic mechanism to allow adequate perfusion of vital organs, including the uteroplacental unit and fetus, and to prepare for the blood loss associated with parturition. ${ }^{60}$ The larger increase of plasma volume relative to erythrocyte volume results in hemodilution. Therefore, a normal pregnancy hematocrit is approximately $32 \%-34 \%$, which is lower than nonpregnant values. ${ }^{61}$

\section{Mean corpuscular volume}

Mean corpuscular volume is a measure of the average erythrocyte size. During pregnancy, there might be a slight increase in mean corpuscular volume, which could prevent the detection of microcytosis associated with iron deficiency. As it is only affected when iron deficiency is pronounced, it does not represent a specific or sensitive measure of body iron stores. ${ }^{32,33}$

\section{Related to fetal growth Fetal growth restriction}

Fetal growth restriction represents pathological inhibition of fetal growth and failure of the fetus to attain its growth potential. ${ }^{61}$ Size at birth can be influenced by factors such as infant gender and parental anthropometric variables, race/ ethnicity, or parity. ${ }^{62}$ Customized birth weight percentiles were designed to better differentiate between infants who are small because in utero growth has been restricted and infants who are small but have reached their individual growth potential. ${ }^{63}$

\section{Small for gestational age}

A small for gestational age baby is defined as a baby whose weight at birth is below the tenth percentile for a given reference growth chart according to their gestational age. It is a proxy measure of fetal growth restriction. ${ }^{62}$ 


\section{Low birth weight}

A low birth weight newborn is one whose weight at birth is $<2500 \mathrm{~g}$. Birth weight is influenced by two major processes: length of gestation and intrauterine growth rate.
Low birth weight is thus caused by a preterm birth (birth at $<37$ weeks of gestation) or restricted fetal growth or a combination of both. ${ }^{64}$

\section{Publish your work in this journal}

Nutrition and Dietary Supplements is an international, peer-reviewed, open access journal focusing on research into nutritional requirements in health and disease, impact on metabolism and the identification and optimal use of dietary strategies and supplements necessary for normal growth and development. The journal welcomes papers covering original research, basic science, clinical \& epidemiological studies, reviews and evaluations, guidelines, expert opinion and commentary, case reports and extended reports. The manuscript management system is completely online and includes a very quick and fair peer-review system, which is all easy to use.

Submit your manuscript here: http://www.dovepress.com/nutrition-and-dietary-supplements-journal 